\title{
Revistas iberoamericanas de comunicación a través de las bases de datos Latindex, Dialnet, DOAJ, Scopus, AHCI, SSCI, REDIB, MIAR, ESCI y Google Scholar Metrics
}

\author{
Rafael Gonzalez-Pardo*, Rafael Repiso**, Jesús Arroyave-Cabrera*** \\ * Universidad del Norte. Colombia. Grupo de investigación: Comunicación y Democracia - Universidad del Tolima. Colombia \\ Correo-e: gonzalezer@uninorte.edu.co | ORCID iD: https://orcid.org/0000-0002-5781-0456 \\ ** Universidad Internacional de La Rioja (UNIR). \\ Correo-e: rafael.repiso@gmail.com | ORCID iD: https://orcid.org/0000-0002-2803-7505 \\ *** Universidad del Norte. Grupo de investigación PBX Comunicación, Cultura y Cambio Social. Colombia \\ Correo-e: jarroyav@uninorte.edu.co | ORCID iD: https://orcid.org/0000-0001-5894-6059
}

Recibido: 08-11-19; 2a versión: 05-01-20; Aceptado: 13-01-20.

Cómo citar este artículo/Citation: González-Pardo, R.; Repiso, R.; Arroyave-Cabrera, J. (2020). Revistas iberoamericanas de comunicación a través de las bases de datos Latindex, Dialnet, DOAJ, Scopus, AHCI, SSCI, REDIB, MIAR, ESCI y Google Scholar Metrics. Revista Española de Documentación Científica, 43 (4), e276. https://doi.org/10.3989/redc.2020.4.1732

Resumen: El presente trabajo es un análisis de las revistas de Comunicación iberoamericanas, sus características y presencias en Latindex, Dialnet, DOAJ, Scopus, AHCI, SSCI, REDIB, MIAR, ESCI y Google Scholar Metrics - GSM. Se analizan las revistas presentes en estos productos, comparando su cobertura, características, representatividad por países, periodicidad de las cabeceras, antigüedad y producción medida en artículos. El objetivo de este trabajo es identificar las revistas científicas de Comunicación en el ámbito iberoamericano para posteriormente estudiar su presencia en las principales bases de datos de revistas. Se analizan elementos sustanciales como la nacionalidad, naturaleza de las instituciones editoras, periodicidad, producción y antigüedad. Se encuentra que la mayor parte de las revistas provienen de instituciones educativas y con una periodicidad semestral. Latindex es la que mayor número de publicaciones tiene seguida por GSM. Las revistas que pertenecen SSCI y Scopus, son las que mayoritariamente están presentes en las bases de datos de revistas.

Palabras clave: revistas científicas; comunicación; Latinoamérica; Iberoamérica; bases de datos bibliográficas; comunicación científica; editoriales científicas; bibliometría.

\section{Ibero American communication magazines through the Latindex, Dialnet, DOAJ, Scopus, AHCI, SSCI, REDIB, MIAR, ESCI and Google Scholar Metrics databases}

Abstract: This article offers an analysis of the Ibero-American Communication journals, their characteristics and presence in Latindex, Dialnet, DOAJ, Scopus, AHCI, SSCI, REDIB, MIAR, ESCI and Google Scholar Metrics - GSM. The journals that belong to these databases are analyzed, comparing their coverage, characteristics, representativeness by countries, periodicity of the headlines, age and production measured in articles. The goal of this work is to identify the scientific communication journals of the Ibero-American area to subsequently study their presence in the main journal databases. Substantial elements such as nationality, nature of the publishing institutions, periodicity, production and seniority are analyzed. It is found that most of the magazines come from educational institutions and their periodicity is by semester. Latindex is the one with the highest number of publications followed by GSM. The journals SSCI and Scopus are those that are mostly present in the journal databases.

Keywords: scientific journals; communication; Latin America; IberoAmerica; databases; scientific communication; scientific publishers; bibliometrics.

Copyright: (c) 2020 CSIC. Este es un artículo de acceso abierto distribuido bajo los términos de la licencia de uso y distribución Creative Commons Reconocimiento 4.0 Internacional (CC BY 4.0). 


\section{INTRODUCCIÓN}

Las revistas científicas se han constituido en uno de los principales vehículos del avance de la teoría y el nuevo conocimiento en una disciplina. De manera colateral, estas publicaciones generan indicadores que son usados para medir los distintos componentes académicos, incluyendo la productividad de las universidades y/o centros de investigación, así como también la de sus docentes e investigadores. Instituciones internacionales como la UNESCO las utilizan como referencia para dar cuenta del avance de la producción científica en los distintos campos del saber en el mundo (WSSR-Unesco, 2015). Si bien, existen posturas críticas frente a este tipo de publicaciones dada su centralidad en el actual ecosistema académico internacional, se hace indispensable explorar con perspectiva crítica su importancia y visibilidad en Iberoamérica en el campo de la Comunicación.

En sus inicios, la mayoría de las publicaciones científicas se dieron en las ciencias duras, desde la aparición en 1665 de las primeras revistas científicas (Journal de Sçavans, Francia y Philosophical Abstracts of the Royal Sociaty, Londres) en áreas tales como las ciencias naturales y las ciencias exactas. Con el avance de las tecnologías las revistas se fueron transformando, incorporando imágenes y gráficos, como fue el caso de Nature en 1869 o migrando al formato digital como ha estado ocurriendo en la actualidad en la mayoría de los campos del conocimiento. Gradualmente, este dominio se extendió a otros campos del saber incluyendo las Ciencias Sociales y las Humanidades, agregando también enfoques de corte cualitativos e interpretativos (Walter, Cody, y Ball-Rokeach, 2018). En el campo de la comunicación, desde mediados del siglo $X X$ se han posicionado revistas que hoy en día abrazan diversos enfoques y abordajes epistemológicos y son referente esencial del campo (Bryant y Miron, 2004).

Si bien esto ha sido considerado un signo positivo, la proliferación de revistas trajo consigo la necesidad de establecer criterios para determinar y diferenciar la calidad de estas publicaciones. Se empezó a relacionar la calidad de las revistas con el prestigio de la editorial, posteriormente con la indexación por parte de revistas de resúmenes (actualmente bases de datos bibliográficas) hasta que Eugene Garfield inventó un indicador científico para medir la calidad de revistas científicas (Repiso, 2015). Uno de los criterios más valorados y extendidos en el mundo académico internacional es precisamente el factor de impacto de Web of Science (WoS) y los indicadores basados en citas.
WoS surgió en Filadelfia, USA, en 1959 por iniciativa del químico y lingüista norteamericano Eugene Garfield. Su nombre original fue Institute for Scientific Information (ISI) y se planteó como objetivo establecer un sistema de indexación basado en las citaciones, concretamente en el indicador Factor de Impacto y la posición que las revistas ocupan en sus categorías temáticas según este indicador. ISI fue vendido a la multinacional Thomson Reuters en los años 90 cuando se creó una plataforma web llamada Web of Science. Posteriormente, Web of Science fue vendida por Thomson Reuters y actualmente pertenece a Clarivate Analytics. Por varias décadas WoS tuvo el monopolio en la medición y evaluación de revistas científicas, hasta que en el 2004 surgió su principal competidor SCOPUS, de la editorial Elsevier.

Web of Science (WoS) y SCOPUS se han posicionado en el contexto internacional como las bases de datos más reconocidas y prestigiosas para revistas en Ciencias Sociales, aunque han sido altamente criticadas por excluir las epistemologías y cosmovisiones del Sur Global (Corona Berkin, 2017). Diversos estudios han mostrado que autores de América Latina, África y países de Europa Central no son citados en las principales revistas de Ciencias Sociales (Alonso Gamboa y Espinosa Reyna, 2015; Beigel, 2013a, 2013b, 2016; Mosbah-Natanson y Gingras, 2015), excluyendo de esta manera una parte sustancial de investigación que no entra en la gran conversación de la circulación del conocimiento internacional. No se puede hablar de conocimiento universal cuando cierta investigación se produce y reproduce en un limitado contexto geográfico.

Beigel (2014) ha cuestionado que ISI por casi 50 años ha servido como una herramienta para promover un cierto prestigio primitivo de la acumulación de las ciencias que ha beneficiado a ciertas áreas geográficas, ciertos idiomas y ciertas disciplinas. "Los circuitos de publicación se han diferenciado por un principio de jerarquía construido con base en recursos institucionales, disciplinas selectas y una proficiencia del inglés" (p. 745). Ortíz (2009) por su parte crítica el hecho que las mediciones de publicaciones e índices de impacto son concebidas como estadísticas universales, pero que son construidas en aquellos centros académicos que están en los más alto del sistema y tiene el poder para establecer las reglas de juego y decidir qué es lo "publicable". Sin embargo, no podemos obviar que la investigación institucionalizada es un fenómeno reciente en los países del "sur" y por tanto ya existe una tradición externa que engloba modelos y sistemas evaluativos. 
En consecuencia, diversas bases de datos de revistas en países iberoamericanos han emergido como respuesta a este domino de los sistemas hegemónicos mundiales de publicación. Entre ellas se destacan Latindex, Dialnet (Difusión de Alertas en la Red), y DOAJ (Directory of Open Access Journals). Así mismo, iniciativas tales como REDIB (La Red Iberoamericana de Innovación y Conocimiento Científico), MIAR (Matriz de Información para el Análisis de Revistas) o Google Scholar Metrics (GSM) son propuestas alternativas a las bases de indexación referenciales que permiten estudiar también publicaciones que no siempre tienen cabida en las bases de datos hegemónicas.

Hasta la actualidad, se han llevado a cabo pocas investigaciones en América Latina que permitan identificar las revistas del Campo de la Comunicación, así como su presencia en bases de datos bibliográficas y características. El estudio comparativo de las revistas sirve de poco si se desconocen las características fundamentales de estas y de los centros y países donde se editan. Los principales aspectos que debemos conocer en una revista es su antigüedad, presencia en bases de datos, periodicidad y tamaño entendido como la producción de artículos. No se puede hablar a la ligera de las revistas españolas de Comunicación en el contexto iberoamericano sin saber que representan el $40 \%$ del total, de igual forma es fundamental conocer el volumen de trabajos que ejecuta cada revista, pues hay revistas que pueden multiplicar por 20 la edición de trabajos de otras revistas menos productivas. Este artículo pretende poner las bases fundamentales sobre las revistas de Comunicación en Iberoamérica para conocer el área de una forma profunda y guiar futuros trabajos.

Con base a la literatura revisada se desprenden las siguientes preguntas de investigación:

1. ¿Cuál ha sido la trayectoria de las revistas iberoamericanas en comunicación a partir de su ubicación geográfica, naturaleza del organismo responsable de su edición, periodicidad de publicación y antigüedad?

2. ¿Cómo ha sido la representatividad y visibilidad de las revistas Iberoamericanas de Comunicación en las bases de datos de revistas?

3. ¿Cómo ha sido la producción documental por parte de las revistas de Comunicación en comparación con las bases de datos de revistas científicas durante el año 2018?

En conclusión, este artículo se centrará en dos aspectos de las revistas iberoamericanas de Comunicación: a) Identificación, representatividad y presencia de las revistas en bases de datos bibliográficas. b) Características editoriales y productivas de las revistas. La siguiente sección ofrecerá un contexto de las principales bases de datos bibliográficas que se van a analizar y los aspectos puntuales que incluiría la investigación.

\section{Bases de datos bibliográficas y revistas ibe- roamericanas}

Quizás la base de datos más reconocida en la academia internacional sea Web of Science. WoS es la plataforma de la empresa Clarivate Analytics que indexa resultados y los clasifica en varias colecciones de bases de datos bibliográficas, a partir de citas y referencias de publicaciones científicas de cualquier disciplina del conocimiento, en ciencia, tecnología, ciencias sociales, artes y humanidades. Dentro de sus bases de datos, para el caso de las Ciencias Sociales se encuentra Social Sciences Citation Index (SSCI), que ejerce hoy una enorme influencia sobre las diversas disciplinas del área. SSCI se ha convertido en una importante herramienta multidisciplinar que pretende aglutinar a las revistas de mayor repercusión con 4.781 cabeceras de 58 categorías temáticas relacionadas con las Ciencias Sociales, una de las cuales es "Communication", y objeto de este estudio. Esta prestigiosa base de datos no ha estado exenta de las críticas por los criterios que utiliza en la inclusión de las revistas (Klein y Chiang, 2004).

Por otro lado, cuenta además con Arts y Humanities Citation Index (AHCI), que pretende registrar las revistas con mayor prestigio y relevancia de esta área del saber. AHCI cuenta con 1828 revistas (año 2019). Sin embargo, en esta base de datos no se realizan métricas de impacto a las revistas, por lo que se les considera de igual calidad a todas. Sin embargo, habría que tener en cuenta su proceso de selección, en el que el factor de las citas no es tan relevante como en las otras dos bases de datos élite de WoS (SSCI y SCIE). (Torre-Espinosa, Repiso, y Montero Díaz, 2019) por lo que la inclusión adolece de elementos de transparencia.

En el año 2015, Web of Science incorporó una nueva base de datos: ESCI (Emerging Sources Citation Index), que incorpora todas las revistas que en la práctica están siendo evaluadas para entrar a formar parte de Web of Science Core Collections, y que responden a un status de las revistas que están "en sala de espera" y que surgen como contrapeso a Scopus (Repiso y Torres, 2016).

Scopus aparece en el año 2004 como el principal competidor de WoS, incorporando en un inicio un mayor número de revistas, lo que permitió la 
presencia más amplia de publicaciones de Latinoamérica. Desde un inició incluyó un mayor número de publicaciones de los países periféricos (Repiso y otros 2017). Desde esta perspectiva, tomaba una clara distancia de WoS, cuya presencia era más amplia en los países anglo-parlantes.

Por otra parte, Google sorprendió en el 2012 con el lanzamiento de Google Scholar Metrics - GSM, la base de datos en la que se encuentra un índice de revistas científicas que son clasificadas por medio de un recuento de citas, buscando entrar en la competencia global con sus similares (WoS y Scopus). GSM clasifica las revistas en función de su índice $\mathrm{h}$. A partir de aquí realiza un ranking de revistas de Comunicación que parte de las citas bibliográficas (Delgado y Repiso, 2013; Repiso y Delgado, 2014). No obstante, GSM también ha sido cuestionado, sus críticas se deben al alcance de su cobertura, el control de las fuentes, la falta de normalización y la consulta de resultados. El grupo EC3 de la Universidad de Granada y concretamente el subgrupo especializado en Google Scholar han llevado a cabo diferentes productos que pretendían ordenar los resultados de Google Scholar Metrics por áreas y años, el más significativo es Journal Scholar Metrics (Martín-Martín y otros, 2017), un ranking mundial de revistas por categorías para las áreas de Humanidades y Ciencias Sociales (9188 revistas), incluyendo a Comunicación. También es destacable que desde la aparición de Google Scholar Metrics este grupo realiza informes anuales sobre la posición de las revistas españolas por áreas (Ayllón, y otros, 2015; Ayllón Millán, y otros, 2016; Ayllón Millán y otros, 2014; Delgado-López-Cózar y Martín-Martín, 2019; Delgado-López-Cózar y otros, 2017; Delgado López-Cózar y otros, 2013a; Delgado López-Cózar y otros, 2013b; Delgado López-Cózar y Martín-Martín, 2018). Uno de los problemas de Google Scholar Metrics es que no podemos conocer los valores de las revistas por años.

En el 2001 nace la Difusión de Alertas en la Red (Dialnet), un portal bibliográfico que surge como un servicio universitario, y que al día de hoy se considera como una hemeroteca virtual con producción científica fundamentalmente hispana. Hoy Dialnet es uno de los mayores portales bibliográficos, y cuya finalidad es la de visibilizar la producción científica a partir del acceso libre a textos completos de literatura científica (Romero Peña y otros, 2015).

La Matriz de Información para el Análisis de Revistas (MIAR) es una iniciativa que surge en cabeza de la Universidad Autónoma de Barcelona desde el año 2003, precisamente como estrategia para la visibilidad de publicaciones en el área de las Humanidades y las Ciencias Sociales, incrementando así el interés de autores y editores de estas áreas. MIAR establece unos criterios de calidad que son fácilmente localizables y constatables, centralizados en una web accesible y usable (Rodríguez-Gairín y otros, 2011). Como punto a tener en cuenta, MIAR utiliza la información que encuentra en las bases de datos de revistas, y desde allí toma en cuenta la pervivencia de las revistas para establecer un Índice Compuesto de Difusión Secundaria, donde se evidencia el alcance internacional (García Aretio, 2017).

Durante el año 2003 se creó por parte de la Budapest Open Access Initiative, una propuesta adoptada por el Open Society Institute (OSI) denominada directorio global y multidisciplinario de revistas científicas, denominado Directory of Open Access Journals (DOAJ). Esta base de datos busca garantizar la visibilidad y acceso a la comunidad académica y científica de los artículos de investigación publicados por revistas arbitradas especializadas, lo cual es un claro indicativo de una mayor visibilidad de sus contenidos. A partir de 2015, DOAJ ha realizado además una selección de los criterios de inclusión y depuración de títulos que no cumplían sus estándares de calidad, robusteciendo la revisión de los documentos por parte de pares (Maldonado, 2017).

En realidad, lo que se hizo fue revisar todas las revistas en Acceso Abierto pues el producto estaba plagado de revistas Predator que estaba perjudicando al prestigio del directorio.

Por su parte, La Red Iberoamericana de Innovación y Conocimiento Científico (REDIB) es otra iniciativa que surgió en 2015 en el encuentro de Rectores Iberoamericanos de Universia, y que en el 2017 se materializó entre la Agencia Estatal Consejo Superior de Investigaciones Científicas (CSIC) y de la red de universidades iberoamericanas Universia (Banco Santander). Esta iniciativa se planteó para facilitar el acceso y la difusión de la producción científica iberoamericana, agrupando un número importante de publicaciones científicas de América Latina. A través de trabajo cooperativo, que es otra de los aportes de esta red, se les ofrecen a los usuarios metadatos del contenido de revistas y artículos, así como también los enlaces directos a las páginas de las revistas allí contenidas (Mazo Flórez, 2017).

Por último, uno de los primeros productos en la geografía iberoamericana es Latindex, que surgió en 1995 en la Universidad Nacional Autónoma de México (UNAM) y se convirtió en una red de cooperación regional a partir de 1997 . Esta base de datos aparece para llenar el vacío de la existencia de un sistema de información propio para las revistas académicas que se producen en América 
Latina y el Caribe, que permitiera a su vez ofrecer instrumentos para la compilación y diseminación de todas las revistas que se editan en la región, así como la definición de criterios e indicadores formales para conocer su calidad (Alonso Gamboa y Espinosa Reyna, 2012).

\section{Las revistas Iberoamericanas de Comunica- ción como objeto de estudio}

Los estudios bibliométricos son cada vez más frecuentes es las diferentes áreas del saber. De esta manera, en campos tales como la Psicología, Buela-Casal y López (2005), revisaron la calidad de la producción científica en este campo, y evidenciaron el aumento del interés en países de Europa y América Latina. En la Educación, Devís- Devís y otros, (2014), evaluaron varias dimensiones estratégicas de ocho revistas iberoamericanas de Educación Física/Ciencias del Deporte presentes en Web of Science, encontrando que la transparencia y la gestión del proceso editorial son mejorables y que la internacionalización y la visibilidad de las revistas son todavía débiles. Finalmente, en Salud, Rodríguez, Ochoa, y Mayta (2014) revisaron el impacto de las revistas de salud colombianas en bases de datos como Publindex, Google Scholar Metrics, Scopus y SciELO, encontrando importantes aspectos diferenciales en la clasificación de las revistas, y recomendando así la clasificación de producción documental con índices internacionales consistentes con los nacionales. Esto demuestra que sobre este tipo de estudios se ha despertado el interés de los investigadores, permitiéndoles así dar cuenta de las particularidades de la investigación, las temáticas e intereses que predominan.

Así mismo, existen algunos artículos de corte bibliométrico o de análisis de contenido sobre el desarrollo de las revistas científicas de la Comunicación, los rankings en las que éstas se encuentran, las estructuras o enfoques de sus artículos, entre otras variables. Tal es el caso de la investigación adelantada por Piñeiro-Naval y Morais (2019), quienes abordaron la producción académica sobre el campo de la Comunicación en España e Hispanoamérica. Por su parte De-Filippo (2013), desarrolló un análisis de la producción científica a partir de las revistas y los documentos publicados en la base de datos Social Science Citation Index (SSCI), evidenciando el importante papel de España como editor de revistas sobre Comunicación (40 del mundo) y como productor de artículos ( $60^{\circ} \mathrm{del}$ mundo), con una notable evolución -en términos cuantitativos- en los últimos cinco años. En el contexto Latinoamericano se analizó la presencia de revistas de Comunicación indizadas en WoS, Scopus y bases de datos de revistas en Acceso Abierto, en donde se evidenció que pese al incremento de la profesionalización e investigación en esta área, la presencia de estas revistas en dichas bases es limitada (Rogel-Salazar y otros, 2017).

En el caso investigaciones enfocadas a la Comunicación desde la producción de revistas científicas, encontramos estudios que se enfocan por un lado, en el desarrollo e impacto de revistas científicas, en particular como Comunicar (PérezRodríguez y otros, 2018), Revista Latina de Comunicación Social en España (Bermejo-Berros, 2014) y El Profesional de la Información - EPI (López -Robles y otros, 2019); la revista brasilera Perspectivas (Pivatto Brum y otros, 2016); Signo y Pensamiento en Colombia (Chaviano, 2007) y Comunicación y Sociedad en México (GómezRodríguez y otros, 2017), entre otras. No obstante, no hay estudios en conjunto de las revistas del área para Iberoamérica.

Por otro lado, aparecen estudios que dan cuenta de la evolución de casos concretos y los dispositivos metodológicos y teóricos de la investigación en Comunicación, a partir del enfoque e impacto específico en algunos países como en España, donde se realizó una caracterización de 25 años de la investigación en Comunicación en revistas (Martínez-Nicolás y otros, 2018). Por su parte en Brasil, se analizaron los principales temas de interés en el campo de la investigación científica dentro del área de la Ciencia de la Información (Liberatore y Herrero-Solana, 2009). En Uruguay se estudió el fenómeno de la Comunicación científica, considerando las publicaciones declaradas en los Currículum Vitae por parte de los investigadores que integran el Sistema Nacional de Investigadores de la Agencia Nacional de Investigación e Innovación de Uruguay (Picco y otros, 2010).

La amplia literatura revisada hasta aquí nos lleva a concluir que no existen estudios longitudinales que permitan constatar de manera integral la presencia y características de las revistas iberoamericanas en el área de la Comunicación en las diversas bases de datos de revistas científicas. Este trabajo pretende arrojar luces en esa dirección, mediante un análisis bibliométrico que permita caracterizar, desde aspectos tales como la representatividad y la visibilidad, las revistas iberoamericanas a partir de sus aspectos editoriales y su productividad.

El objetivo de este trabajo por tanto es el de identificar las revistas iberoamericanas de Comunicación, su presencia en las principales bases de datos de revistas, su distribución por países, naturaleza del organismo editor y sus características según periodicidad, antigüedad y producción. 


\section{MATERIAL Y MÉTODOS}

El presente trabajo es un análisis bibliométrico de la presencia y características generales de las revistas Iberoamericanas de Comunicación incluidas en un corpus de 10 bases de datos bibliográficas: Web of Science: Social Sciences Citation Index (SSCI), Arts and Humanities Citation Index (AHCI) y Emerging Sources Citation Index (ESCI); Scopus; Google Scholar Metrics, Latindex, DOAJ, MIAR, Dialnet y Redib.

Se entiende la región Iberoamericana como el conjunto de países americanos que fueron colonizados por parte de los países incluidos en la península más occidental de Europa, y tienen el castellano o el portugués como idiomas oficiales. Se determina en este sentido que se tomarán los países de la región Sudamericana: Argentina, Bolivia, Brasil, Colombia, Chile, Ecuador, Paraguay, Perú, Uruguay y Venezuela; Europa: España y Portugal; Centroamérica y el Caribe: Costa Rica, Cuba, República Dominicana, El Salvador, Guatemala, Nicaragua, Panamá y Puerto Rico; y América del Norte: México.

\subsection{Muestra}

Para la identificación de las revistas de Comunicación Iberoamericanas, se definieron para cada una de las bases de datos de revistas criterios de inclusión y de exclusión, arrojando una muestra inicial de 669 revistas. Sin embargo, esta cifra se redujo a 297 al eliminar duplicados y revistas que no eran propiamente de Comunicación. Se han considerado como revistas de Comunicación las pertenecientes a una categoría de Comunicación de las bases de datos estudiadas o la etiquetación como tal, y que se encontraban en el área temática: Ciencias de la Comunicación y la Cultura; Ciencias Sociales y Humanidades; Artes y Humanidades. En el caso de Google Scholar Metrics se buscaron términos relativos al área en el buscador interno (Periodismo, Comunicación, Cine, Publicidad, Relaciones Internacionales, etc.), se comprobó la presencia de las revistas identificadas en las otras bases de datos y los listados de revistas de Comunicación de Google Scholar publicados por Delgado y Repiso (2013) y Repiso Caballero y Delgado López-Cózar (2014). La búsqueda y carga de datos se llevó a cabo en septiembre y agosto de 2019.

Así mismo, teniendo en cuenta los criterios de exclusión, que estaban enmarcados por aquellas revistas que en los resultados aparecen que no están en circulación; revistas que, si bien hacen parte del el área temática de la base de datos o en la categoría, sus contenidos están más enfocados a otras áreas del conocimiento, para ello se ha revisado el ámbito de la revista y los últimos números publicados. De las 61 excluidas, están por ejemplo revistas como Audiology-communication Research de Brasil, Revista internacional del libro digitalización archivos y bibliotecas de España, Revista Onis Ciência de Portugal, entre otras. También se excluyeron aquellas revistas "irregulares" (aquellas que han dejado de publicar periódicamente sus números en sus webs site, desde 2018). En total se excluyeron 35 revistas, dentro de las que se encuentran revistas como Temas y Problemas de Comunicación de Argentina, Formats de España y Formats de Venezuela, entre otras.

Se retiraron de acuerdo a lo anterior un total de 97 revistas de la muestra (41 de España, 19 de Brasil, 13 de Argentina, 7 de Chile, 5 de Venezuela, 3 de México, 3 de Ecuador y 1 de Perú, Guatemala y Colombia, respectivamente). Estas revistas fueron revisadas por separado por dos de los tres autores del documento para validar la selección, coincidiendo al $100 \%$ en el listado propuesto para exclusión. La muestra final que se estudia es de 200 revistas en las 10 bases de datos seleccionadas.

\subsection{Procedimiento}

Se han analizado los aspectos recogidos en la tabla I: Representatividad, visibilidad, distribución por países, tipología institucional, periodicidad, antigüedad y producción. Los datos han sido tratados y visualizados con Microsoft Excel, Tableau (Murray y Chabot, 2013) y de diagramas de Veen (http:// www.interactivenn.net).

\section{RESULTADOS}

\subsection{Trayectoria de las revistas iberoamerica- nas en comunicación}

El presente trabajo identifica 200 revistas científicas de Comunicación en el contexto iberoamericano, con presencia en 13 de los 22 países estudiados (Brasil, Argentina, Colombia, Chile, Ecuador, Uruguay, Perú, Bolivia, Venezuela, España, Portugal, Cuba y México). Hay que indicar que muchas de estas revistas también publican sobre temas de otras áreas del conocimiento como Educación, Filosofía o Sociología. Por ejemplo, luego del proceso de depuración, Latindex, la base de datos en la que se encuentra el mayor número de revistas, pasó de 156 revistas a 132 .

Un aspecto coincidente en las revistas que hacen parte de la mayoría de las bases de datos de revistas es la naturaleza de sus editores (figura 1). La mayor parte de las revistas de investigación en Comunicación Iberoamericanas provienen de institu- 
Tabla I. Elementos analizados en las revistas de Comunicación Iberoamericanas en bases de datos bibliográficas

\begin{tabular}{|c|c|}
\hline \multicolumn{2}{|r|}{ Representatividad y visibilidad de las revistas } \\
\hline $\begin{array}{l}\text { Presencia de revistas } \\
\text { Iberoamericanas de } \\
\text { Comunicación según bases de } \\
\text { datos producción científica }\end{array}$ & $\begin{array}{l}\text { Se busca analizar la presencia y difusión de las revistas analizadas en las siguientes } \\
\text { bases de datos: } 1 \text {. Social Sciences Citation Index (SSCI). 2. Arts and Humanities } \\
\text { Citation Index (AHCI). 3. Emerging Sources Citation Index (ESCI). 4. Scopus. } \\
5 . \text { Google Scholar Metrics (GSM). 6.Latindex. 7.DOAJ. 8. MIAR 9.Dialnet. 10.Redib. } \\
\text { (Figuras 4a y 4b). }\end{array}$ \\
\hline Nacionalidad de las revistas & $\begin{array}{l}\text { Se analizará la distribución por países de las revistas Iberoamericanas de } \\
\text { Comunicación en las bases de datos de revistas. La nacionalidad de las revistas se } \\
\text { asigna en el momento de solicitar el ISSN y no se puede cambiar sin cambiar de } \\
\text { ISSN. (Tabla III). }\end{array}$ \\
\hline \multicolumn{2}{|r|}{ Propiedad, periodicidad y producción } \\
\hline $\begin{array}{l}\text { Naturaleza del Organismo } \\
\text { responsable }\end{array}$ & $\begin{array}{l}\text { Se utiliza la siguiente clasificación de tipologías de editoriales a. Asociación científica } \\
\text { o profesional. b. Universidad. c. Editorial. d. Centro de estudios. e. Instituto. f. } \\
\text { Grupo. e. Federación. g. Otros. (Figura 1). }\end{array}$ \\
\hline Periodicidad & $\begin{array}{l}\text { Los datos de periodicidad fueron consultados en las bases de datos que contaban } \\
\text { con esta información; sin embargo, se realizó una verificación en los sitios web de } \\
\text { cada una de las revistas, pues no había coincidencia con lo encontrado en productos } \\
\text { como Latindex (pues muchas revistas han cambiado con el tiempo). Las variables } \\
\text { son: Permanente, mensual, bimestral, trimestral, cuatrimestral, semestral y anual. } \\
\text { (Figura } 2 \text { y tablaII). }\end{array}$ \\
\hline Antigüedad & $\begin{array}{l}\text { Se estudia la antigüedad de las revistas por grupos en relación a su fecha exacta } \\
\text { de creación y se comparan a partir de las bases de datos bibliográficas donde se } \\
\text { encuentran las revistas de Comunicación Iberoamericanas, las bases de datos están } \\
\text { ordenadas según el promedio (Figura 3). }\end{array}$ \\
\hline Producción & $\begin{array}{l}\text { Se analiza la producción de trabajos científicos del año } 2018 \text { (puesto que } 2019 \text { está } \\
\text { en desarrollo), las bases de datos están ordenadas por el promedio de los valores } \\
\text { (Figura 5). }\end{array}$ \\
\hline
\end{tabular}

ciones educativas (149), pues son mayoritariamente editadas por universidades $(74,5 \%)$, seguidos por Asociaciones ( $8 \%$ ) y Editoriales (5.5\%). Estas últimas son en su mayoría producidas en Europa (10). Tan solo una sola revista es producida por casas editoriales en Latinoamérica (Revista Cine Documental de Argentina). En la categoría otros, se encuentran aquellas publicaciones que son realizadas por entidades que apenas tienen representación como Escuelas, Redes, Observatorios, Organizaciones privadas y Laboratorios. Las universidades que más revistas de Comunicación editan son la Universidad Nacional de La Plata (10 revistas), Universidad Complutense de Madrid (9 revistas) y Universidad de Sao Paulo (4 revistas). Otro elemento para destacar es que el total de las revistas que se analizaron son de acceso abierto, sólo una, El Profesional de la Información, ofrece contenidos por pago.

Otro aspecto indicativo y característico de las revistas Iberoamericanas es su periodicidad. La gran mayoría de las revistas encontradas en las 10 bases de datos estudiadas tienen aparición semestral (figura 2). Hay un bajo número de revistas que son publicadas de manera permanente o bimestral especialmente por España. Dentro de las publicaciones trimestrales, la mayoría se concentran en Europa (11) y de Sudamérica sobresale Brasil (5). Las revistas con publicación cuatrimestral se concentran entre España (34) y Brasil (32). Anualmente, España es el país que más revistas concentra (24). En las demás regiones, particularmente en Sudamérica, la publicación es semestral (68 revistas) seguido por España (34 Revistas).

El análisis de periodicidad (tabla II), en la que se resaltan en cada una de las bases de datos estudiadas la periodicidad de mayor impacto, evidencia grandes diferencias, particularmente entre las revistas de élite. Las revistas Iberoamericanas en Comunicación son publicadas semestralmente a diferencia de SSCI, que se centra en publicaciones bimestrales y trimestrales; las revistas de $\mathrm{AHCI}$ son por el contrario semestrales. Con menor presencia, Scopus cuenta con revistas trimestrales; sin embargo, el principal elemento a analizar es que 9 de los 10 productos cuentan con revistas cuya mayor representatividad tienen publicación semestral, seguidas por las publicaciones anuales y las cuatrimestrales. En general, se puede afirmar que las bases de datos bibliográficas cuanto menos exigente son en su indexación tienen periodos de publicación más largos, como el caso de Latindex, GSM, DOAJ, MIAR y ESCI. 
Figura 1. Naturaleza de los editores revistas de Comunicación Iberoamericanas

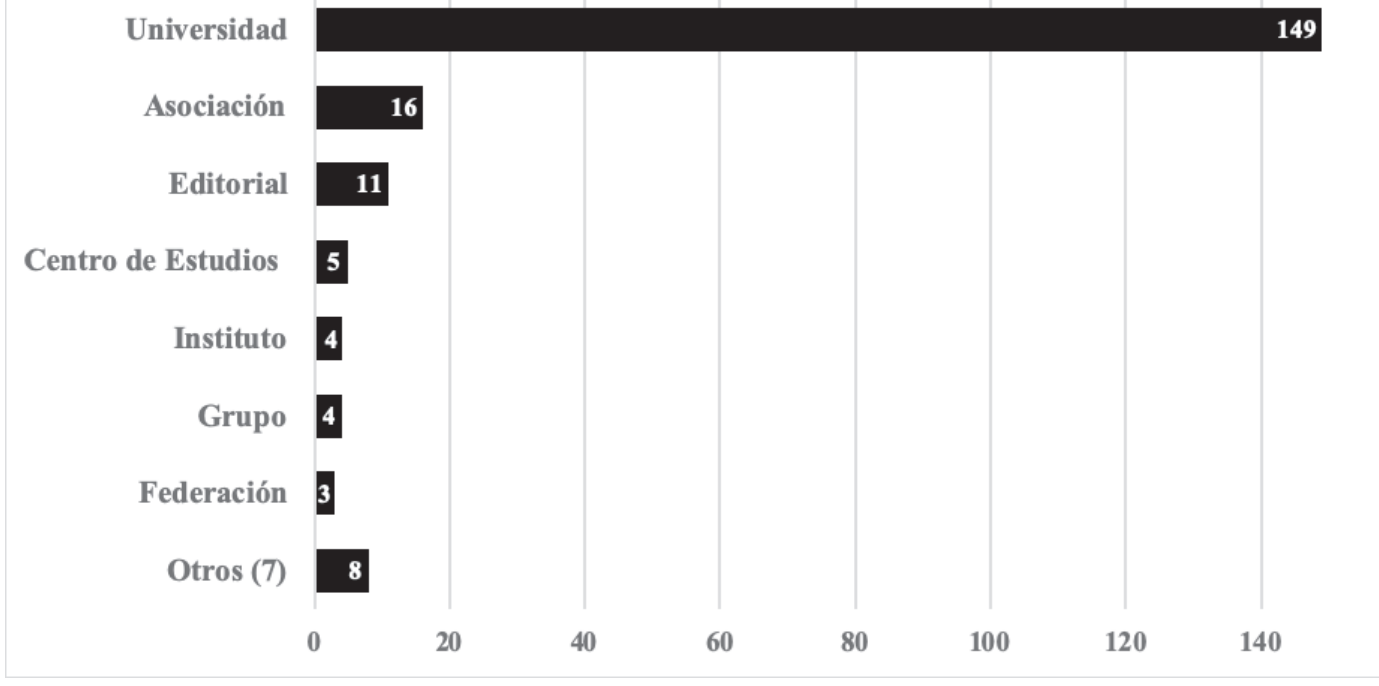

Figura 2. Periodicidad de las revistas Iberoamericanas de Comunicación

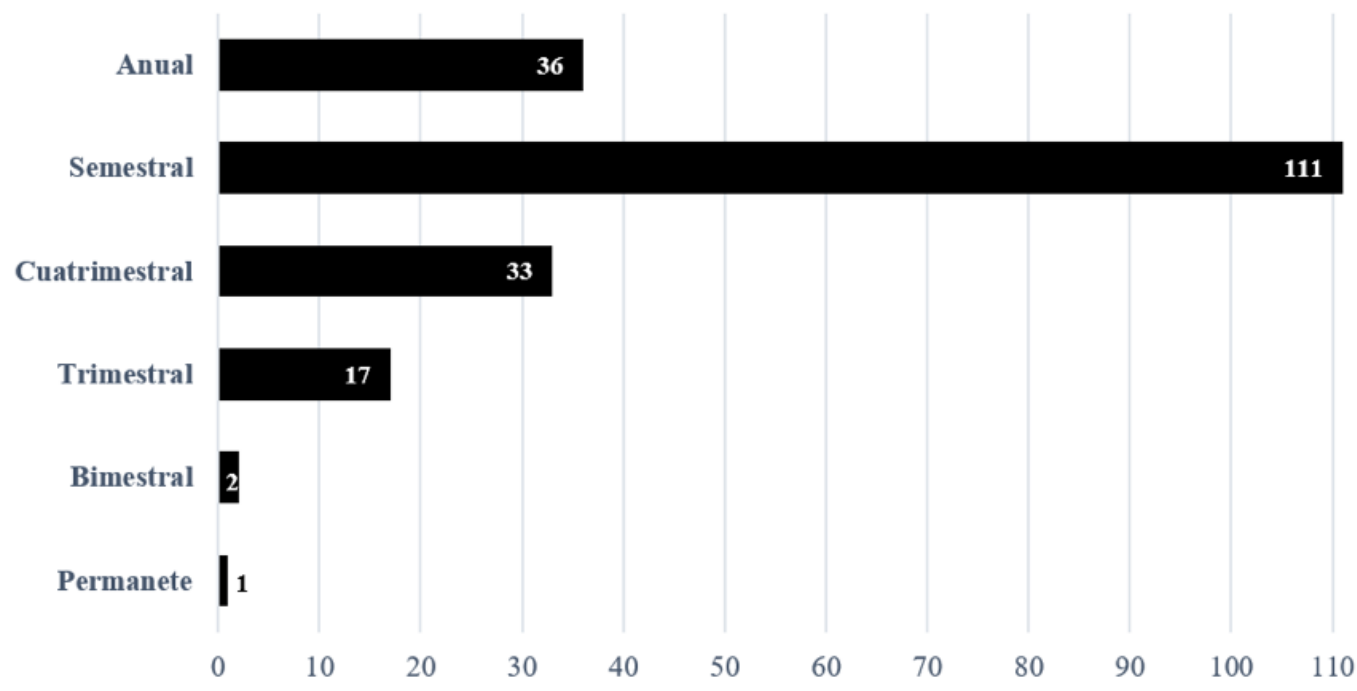

La antigüedad de las publicaciones periódicas de Comunicación es otro elemento que nos permite diferenciar los conjuntos de revistas agrupados en las diferentes bases de datos analizadas (figura $\mathbf{3}$ ). Curiosamente, de las revistas más antiguas tales como E-Compos y Vozes e Diálogo de Brasil, Anagramas de Colombia, Contratexto de Perú; y Revista de comunicação e linguagens de Portugal no están dentro de las bases de datos élite; tan solo Anàlisi: Quaderns de Comunicació i Cultura de España, y Comunicación y Sociedad de México, se encuentran en Scopus. Además, otro hallazgo, es que el conjunto de las publicaciones de SSCI, AHCI y Scopus, donde además pertenecen revistas que ya estaban en circulación antes de que entrara en vigencia, tienen una media entre 21 y 22 años, por encima del resto.

En cambio, en bases de datos como DOAJ, Latindex, Redib, MIAR y Dialnet la media oscila entre 12 y 13 años. Dentro de estas bases de datos también se encuentran las revistas más antiguas. GSM y DIALNET y ESCI son las bases en la que aparecen las revistas más jóvenes, incluso con tan solo un año de antigüedad en el caso de Dialnet (2 revistas); DOAJ, Latindex y nuevamente GSM aparecen revistas con dos años de antigüedad (9 revistas). Un tercio del total de las revistas $(33,5 \%)$ tienen ocho o menos años de antigüedad. 
Tabla II. Periodicidad de las revistas Iberoamericanas de Comunicación en bases de datos de revistas científicas

\begin{tabular}{|c|c|c|c|c|c|c|c|c|c|c|}
\cline { 2 - 13 } & Latindex & Dialnet & DOAJ & REDI & MIAR & ESCI & Scopus & SSCI & A HCl & GSM \\
\hline Permanete & 1 & 1 & 1 & 1 & 1 & 1 & 1 & 0 & 0 & 1 \\
\hline Bimestral & 1 & 1 & 1 & 0 & 1 & 0 & 1 & $\mathbf{1}$ & 0 & 2 \\
\hline Trimestral & 11 & 6 & 10 & 4 & 10 & 4 & 7 & $\mathbf{1}$ & 0 & 16 \\
\hline Cuatrimestral & 16 & 5 & 16 & 9 & 14 & 8 & 6 & 0 & 0 & 22 \\
\hline Semestral & $\mathbf{8 2}$ & $\mathbf{3 5}$ & $\mathbf{5 7}$ & $\mathbf{1 9}$ & $\mathbf{4 8}$ & $\mathbf{1 9}$ & $\mathbf{1 2}$ & 0 & $\mathbf{2}$ & $\mathbf{6 8}$ \\
\hline Anual & 21 & 13 & 14 & 6 & 14 & 8 & 2 & 0 & 0 & 10 \\
\hline
\end{tabular}

Figura 3. Antigüedad de las revistas de Comunicación Iberoamericanas en comparación con las bases de datos de revistas científicas

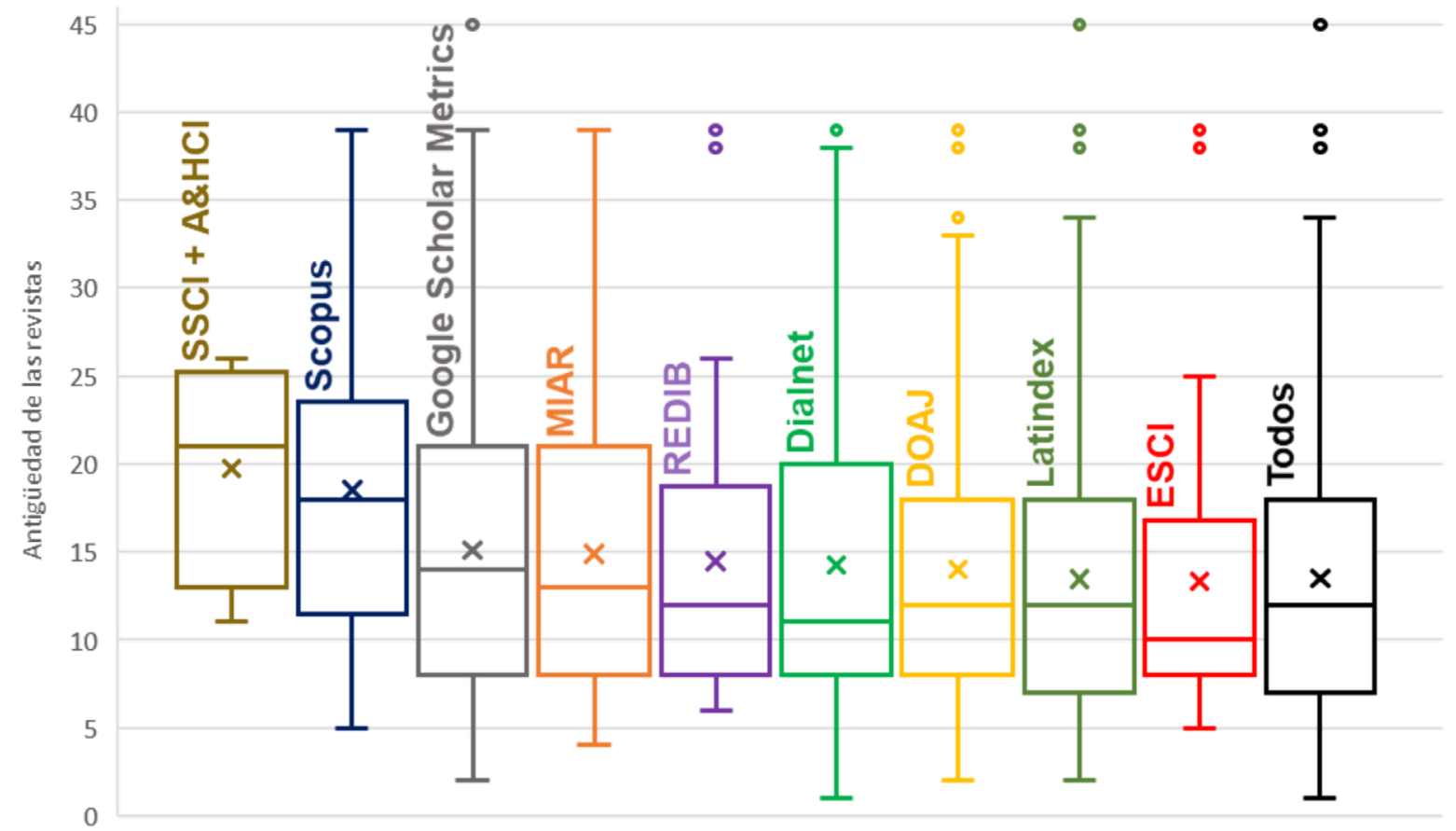

\subsection{Representatividad y visibilidad de las re- vistas Iberoamericanas de Comunicación}

La base de datos que presenta un mayor número de estas revistas es Latindex que aglutina al 66\% de la población (132), seguida de Google Scholar Google Scholar reporta el 59,5\% (119); DOAJ el 49,5\% (99); MIAR el 44\% (88); Dialnet el 31\% (61); ESCI el 20\% (40); Redib el 19,5 (39); Scopus el $14,5 \%$ (29) y AHCI (2) SSCI (2) solamente el $1 \%$.

Si se estudia la presencia de este conjunto de revistas de Comunicación en las bases de datos de Web of Science, Scopus y Google Scholar (Figura 4a), vemos como menos de la mitad de las revistas de Scopus (13) aparecen en Web of Science (SSCI
+ AyHCI + ESCI), aunque Scopus sí coincide en indexar las cuatro revistas que se encuentran en SSCI y AHCI, por lo que el elemento diferencial son las revistas emergentes. Por otro lado, si estudiamos el conjunto de la figura $\mathbf{1} \mathbf{b}$, que contrasta las bases de datos iberoamericanas Latindex y Dialnet con los productos DOAJ, MIAR y Google Scholar, 13 revistas confluyen en las cinco bases de datos, siendo Latindex la que cuenta con el mayor número de revistas que coinciden con otros productos. DOAJ por su parte, también cuenta con un número importante de revistas que se interseccionan con otras bases de datos, pues 11 de ellas aparecen en 4 de las 5 bases de datos con mayor muestra representativa. Diecinueve revistas confluyen con tres bases de datos distintas. 
Analizando las bases de datos que registran el impacto de citas de los artículos, es GSM la que mayor número de revistas tiene seguido por ESCI. Así mismo, Latindex es la base de datos en general que mayor número de publicaciones tiene, evidenciando además que las 132 revistas son de acceso abierto.

Se observa que las revistas que están en las bases de datos más exigentes (SSCI y Scopus), son las que mayor presencia tienen en la totalidad de las bases de datos. En efecto, las revistas que pertenecen a las bases de datos de alto impacto son las que mayoritariamente están presentes en el resto.

La distribución comparada por países en las 10 bases de datos estudiadas (Tabla III), demuestra cómo Latindex presenta la mayor cobertura por países seguido por GSM, DOAJ y MIAR. España, es el país con mayor representatividad en la mayoría de las bases de datos y el único que cuenta con publicaciones en SCSI y AHCI. En el caso sudamericano, Brasil cuenta con mayor número de revistas, seguido, con una gran diferencia, por Argentina y Colombia. Colombia por su parte cuenta con el mismo número de publicaciones de Portugal. Cuba, el único país de Centro América y el Caribe reportado en las bases, es el de menor presencia, con un número de publicaciones igual al de Bolivia.

Frente a las bases de datos más exclusivas, si se suman la totalidad de las revistas de SSCI, AHCI y ESCI, existe un mayor número de publicaciones en WoS comparado con Scopus. Solamente ESCI es un $5,5 \%$ más amplia que Scopus (11 revistas), y en total WoS supera Scopus por 15 publicaciones, es decir WOS es un más grande que Scopus en un $51 \%$.
Llama la atención la acumulación de revistas de comunicación que poseen España (69) y Brasil (65). Países como México, que tradicionalmente ha sido referenciado como un centro difusor y exportador de investigación en Comunicación no cuenta con un gran protagonismo. Por otro lado, Argentina es el tercer país con mayor número de revistas en las bases de datos analizadas. Si se reflexiona este resultado a partir de las bases de datos de alto impacto, Colombia y Chile tienen un mejor desempeño, pues cuentan con un número menor de publicaciones, pero que hacen parte de Scopus y ESCI.

\subsection{Producción de trabajos científicos de las revistas iberoamericanas durante el año 2018}

Al analizar los diferentes conjuntos por su producción medida en el número de artículos publicados en el 2018 (figura 5), se detecta que al igual de lo que sucede con la antigüedad de las revistas, se presenta una diferencia fundamental: Las revistas que se encuentran en las bases de datos de WOS (SSCI-AHCI) son las que más artículos publican, a gran distancia del resto. Sucede el mismo fenómeno con las revistas que se encuentran en Scopus, pues existe mayor producción documental, con una media de 45 documentos. Hay un grupo de 19 revistas que publican 8 o menos artículos al año y a su vez estas se encuentran indexadas en MIAR, Latindex, DOAJ, ESCI y GSM. Dialnet es la base de datos donde se evidencian revistas que producen la media más baja de documentos: 20 .

Figura 4. Diagramas de Venn sobre la presencia de revistas Iberoamericanas de Comunicación en bases de datos bibliográficas

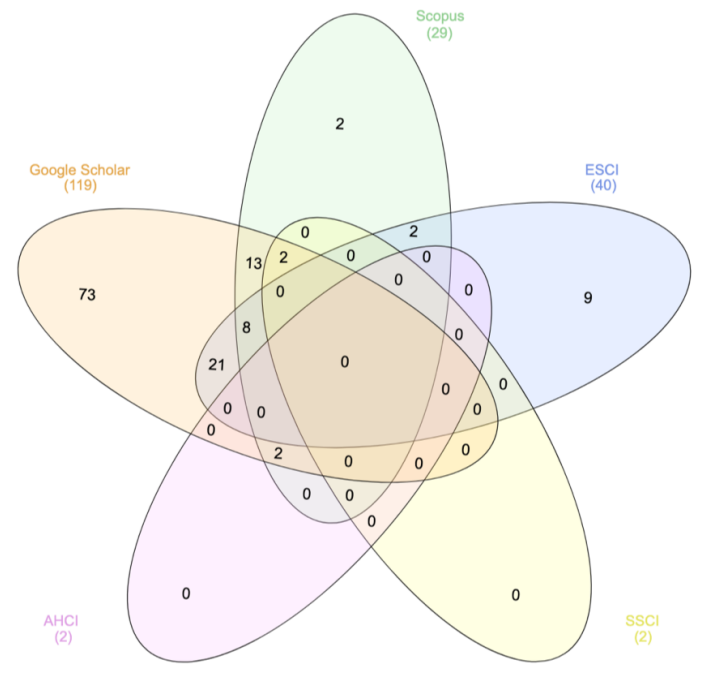

Figura a

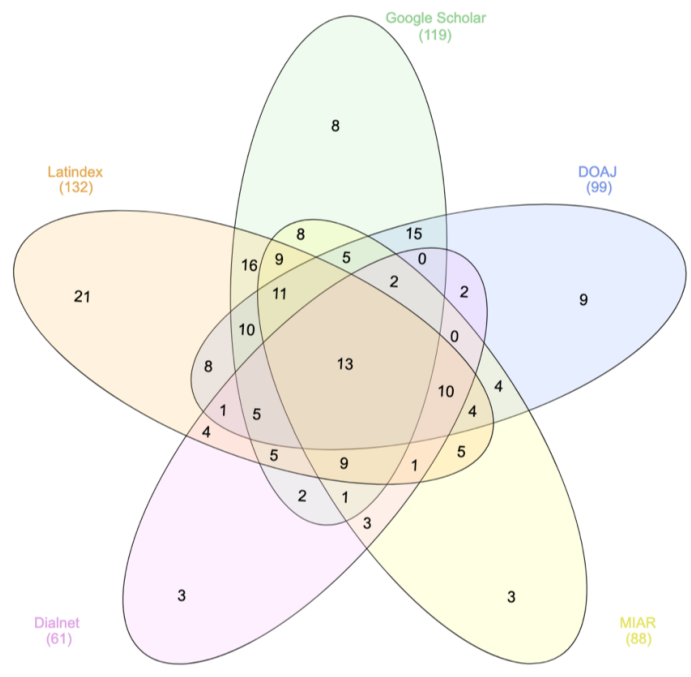

Figura b 
Tabla III. Distribución por países de revistas Iberoamericanas de Comunicación en bases de datos científicas

\begin{tabular}{|c|c|c|c|c|c|c|c|c|c|c|c|}
\hline & Total & Latindex & GSM & DOAJ & MIAR & Dialnet & ESCI & REDIB & SCOPUS & SCSI & AHCI \\
\hline España & 69 & 51 & 44 & 29 & 43 & 34 & 23 & 23 & 11 & 2 & 2 \\
\hline Brasil & $\overline{63}$ & 31 & 43 & 36 & 15 & 0 & 4 & 4 & 7 & 0 & 0 \\
\hline Argentina & 17 & 17 & 5 & 7 & 4 & 6 & 4 & 2 & 0 & 0 & 0 \\
\hline Portugal & 14 & 12 & 9 & 7 & 8 & 1 & 2 & 2 & 4 & 0 & 0 \\
\hline Colombia & 11 & 7 & 7 & 6 & 6 & 8 & 1 & 2 & 2 & 0 & 0 \\
\hline Chile & 7 & 5 & 3 & 3 & 3 & 4 & 3 & 3 & 2 & 0 & 0 \\
\hline México & 4 & 1 & 2 & 1 & 3 & 1 & 0 & 0 & 1 & 0 & 0 \\
\hline Perú & 4 & 2 & 1 & 3 & 3 & 2 & 1 & 0 & 1 & 0 & 0 \\
\hline Venezuela & 3 & 1 & 2 & 1 & 0 & 2 & 0 & 0 & 0 & 0 & 0 \\
\hline Ecuador & 3 & 1 & 2 & 3 & 1 & 1 & 1 & 1 & 1 & 0 & 0 \\
\hline Uruguay & 2 & 2 & 1 & 2 & 2 & 1 & 1 & 1 & 0 & 0 & 0 \\
\hline Bolivia & 2 & 2 & 0 & 0 & 0 & 0 & 0 & 0 & 0 & 0 & 0 \\
\hline Cuba & 1 & 0 & 0 & 1 & 0 & 1 & 0 & 0 & 0 & 0 & 0 \\
\hline & & 132 & 119 & 99 & 88 & 61 & 40 & 38 & 29 & 2 & 2 \\
\hline
\end{tabular}

Frente a las la revistas que más producen artículos en la totalidad de las bases de datos estudiadas, sobresalen 6 que en el año 2018 tienen más de 100 documentos y que están presentes en 3 de la totalidad de las bases de datos analizadas: La revista Observatorio de Brasil, que es la que más documentos publicó (203), Razón y Palabra de Ecuador (139) e Intexto de Brasil (138). Question de Argentina que está en cuatro bases de datos, incluidas ESCI y es una de las más jóvenes de la muestra total, desarrolló 121 documentos.

Por su parte, al analizar los resultados en Google Scholar Metrics, se encontraron revistas con menos de 20 artículos durante el 2018, lo que es un indicio de que en principio pueden no cumplir el requisito de publicar 100 trabajos en 5 años. Se resaltan las revistas españolas: Cic Cuadernos de Información y Comunicación, Comunicación; revista Internacional de Comunicación Audiovisual, Publicidad y Estudios Culturales y Scire: representación y organización del conocimiento, cada una con 14 documentos en el 2018. También Secuencias: Revista de Historia del Cine, IC Revista Científica de Información y Comunicación, cada una con 15 documentos; Anàlisi: Quaderns de Comunicació i Cultura y Sphera publica con 16 documentos; finalmente, Telos: Cuadernos de comunicación e innovación con 17 documentos.

De Colombia aparecen Signo y pensamiento y Nexus Comunicación, cada una con 15 documentos, además de la revista Luciérnaga con 18 documentos. Brasil aparece con las revistas Informação y Sociedade y Ação Midiática - Estudos em Comunicação, Sociedade e Cultura, cada una con 17 documentos. Finalmente, las portuguesas
Estudos de Jornalismo y Prisma.com con 17 documentos; la mexicana Ricsh con 19 documentos y la chilena F@ro con 16.

\section{DISCUSIÓN Y CONCLUSIONES}

Como primera medida, se determinó que Latindex se posiciona como la base de datos donde las revistas de iberoamericanas de Comunicación tienen mayor presencia, conteniendo dentro de su sistema un total de 132 revistas científicas, superando a GSM, DOAJ y MIAR que le siguen en la lista. Latindex además es la única base de datos que integra dentro de sus publicaciones la producción científica de Bolivia con 2 revistas evidenciadas. En la búsqueda de desarrollar un sistema de información para las publicaciones científicas de América Latina -que más adelante contó con la integración de España y Portugal-, Latindex difunde y visibiliza los trabajos académicos principalmente de España, Brasil, Argentina y Colombia, respectivamente. Sin embargo, los países de América Latina y el Caribe cuentan con una baja distribución de trabajos publicados en las bases de datos analizadas en comparación con España y Portugal. Cuba, por ejemplo, es el único país de Centro América y el Caribe que se encuentra dentro de la distribución de Revistas Iberoamericanas de Comunicación en las bases de datos elegidas, pero es el de menor presencia junto con Bolivia.

Brasil es el país latinoamericano con mayor presencia de revistas de Comunicación en las bases de datos elegidas para realizar el análisis bibliométrico (63), muy cercano a España, que es el que mayor presencia tiene (69). Esto lo que permite eviden- 
Figura 5. Producción documental por parte de las revistas de Comunicación en comparación con las bases de datos de revistas científicas durante el año 2018

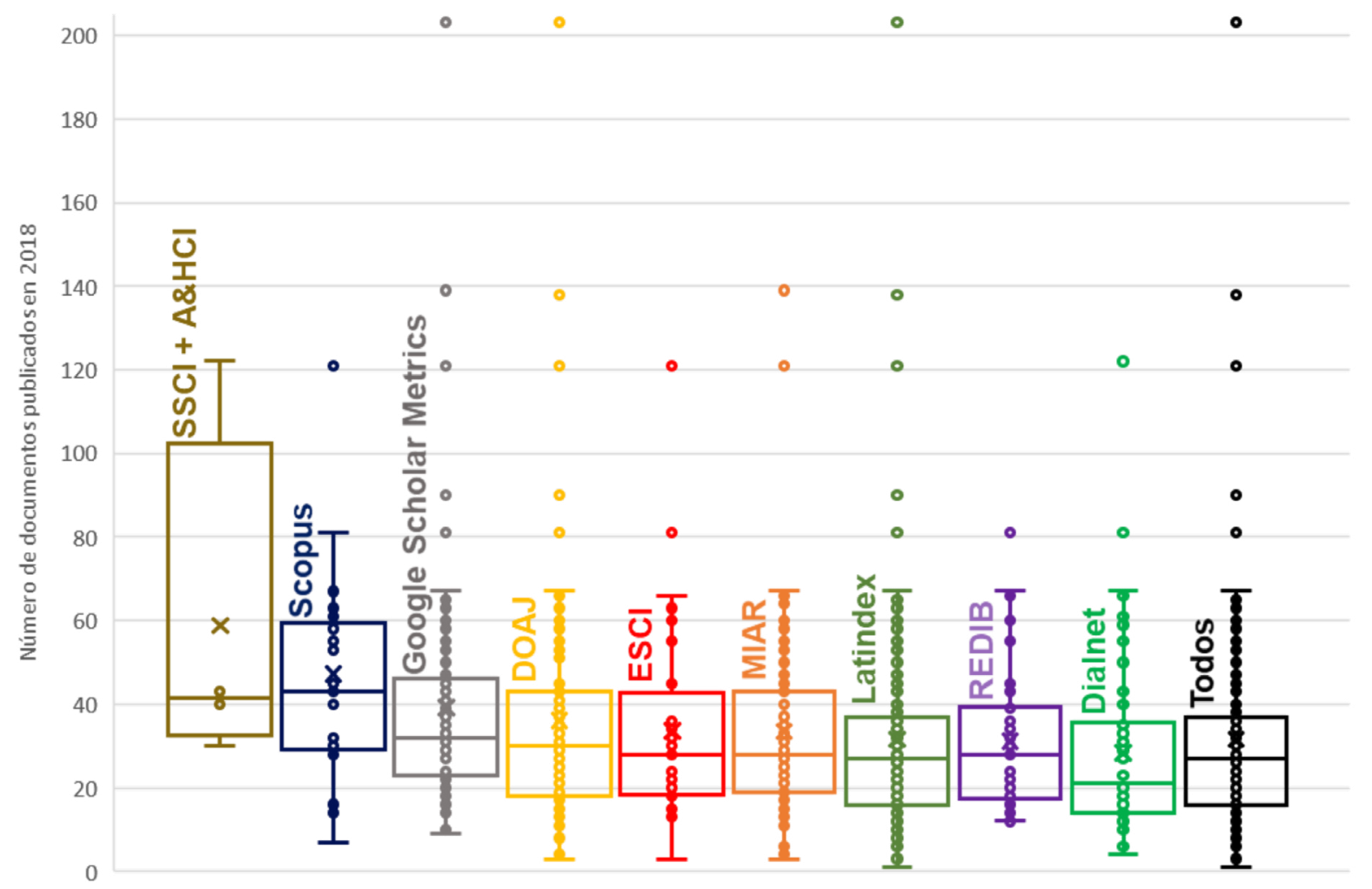

ciar es que en estos dos países se concentra gran parte de las revistas que están en la mayoría de los productos analizados, pues ocupan un $66 \%$ de la muestra total. Si bien Brasil es el país de América Latina con mayor presencia en las bases de datos, es importante analizar estos resultados a luz de los indicadores poblacionales, pues este país actualmente cuenta con 208,5 millones de personas, que si se compara con Argentina (45,39 millones), que es el segundo país con mayor presencia, hay una diferencia de más de 163 millones de habitantes, y con Colombia (45 millones), una cifra similar: 163,5 millones de habitantes. Siguiendo con este análisis, Uruguay cuenta con una baja presencia de publicaciones, pero es el país con el menor número de habitantes (3,5 millones) aunque sobresale por tener un elevado número de centros universitarios, en total 40, según el Ranking web of Universities (Webometrics).

La naturaleza de la edición de Revistas Iberoamericanas de Comunicación se concentra en mayor medida en instituciones educativas. Por su parte, las asociaciones y federaciones se encuentran en los últimos lugares, considerándose así un índice que refleja la ausencia de trabajos científicos por parte de editores diferentes al ámbito educativo como centros de estudio, grupos, editoriales o institutos. Frente a esta variable, también se halló que son las revistas de Europa las que cuentan prácticamente de manera exclusiva con realización de publicaciones por parte de editoriales privadas. Lo que es normal en otros países, donde la naturaleza de los editores recae en las Asociaciones y en organizaciones privadas, en América Latina, por el contrario, recae sobre las Universidades. Frente a esto hay que anotar que las empresas privadas profesionalizan más el sistema de producción editorial, pues a diferencia de las revistas producidas por las instituciones universitarias, se puede evidenciar una clara remuneración o consideración especial a los editores.

Es deseable que las universidades cuenten con revistas científicas autónomas, y para ello se necesita contar con soporte editorial y recursos humanos (masa crítica de investigadores especializados), que se destaquen en el área temática desarrollada; o deben tener al menos capacidad para atraerlos (Repiso y otros, 2019). Si bien las Universidades cada vez más dentro de sus indicadores giran la mirada a contar con una mayor presencia en 
revistas científicas, existen otros objetivos misionales, que hacen que no sea la edición de revistas científicas objeto de prioridad para estas instituciones. Además, tampoco se destina exclusivamente el presupuesto para su robustecimiento. Esto a diferencia de las editoriales privadas, en donde las revistas lo son todo.

Otro punto que fortalecer es el papel de las Asociaciones de Comunicación Iberoamérica, en donde la Federación Latinoamericana de Facultades de Comunicación - FELAFACS a través de su revista Diálogos apenas tiene presencia en bases de datos de revistas. En los países en los que se encontraron revistas editadas por asociaciones, la gran mayoría está presente en las bases de datos de corte Latinoamericano y que no tienen alto nivel de exigencia. Por ejemplo, en Brasil, de las 18 encontradas, tan solo 2 pertenecen a Scopus: Informação y Sociedade e Interface Comunicacao, Saude E Educacao. En España, por ejemplo, una pertenece a ESCI Comunicación: Revista de Recerca i d'Anàlisi y otra a Scopus: Signa, la revista de la Asociación Española de Semiótica. Si bien, en países como Estados Unidos, en donde existen entidades con un claro músculo financiero, como es el caso de la International Communication Association - ICA, que hoy cuenta con 5 revistas en las bases de datos de revistas más exclusivas, los países de Iberoamérica podrían utilizar como estrategia mejorar su nivel asociativo y desde las actuales asociaciones, trabajar por el posicionamiento de las publicaciones iberoamericanas en las bases de datos bibliográficas regionales e internacionales.

En relación con la periodicidad, los resultados muestran que la periodicidad promedio de las revistas es lenta. La muestra elegida para este estudio refleja que las publicaciones de las revistas se realizan de manera semestral mayoritariamente. Pocas son las revistas que editan números permanentemente, a diferencia de una pequeña representación de producciones que cuentan con menor tiempo de periodicidad, que fundamentalmente se refiere a publicaciones españolas. La velocidad en los procesos de edición científica es fundamental para el desarrollo de la investigación, pero en los resultados se evidencia que, en la región iberoamericana, la periodicidad permanente no está presente, pues sobresalen fundamentalmente en las bases de datos estudiadas las publicaciones anuales y semestrales. Esto obedece quizás a dos razones: (1) Por un lado, no reciben los suficientes manuscritos para publicarse de manera permanente. (2) No tienen energía, es decir no están lo suficientemente profesionalizadas para sacar varios números o ediciones de manera permanente. Se observó que, teniendo en cuenta los resultados hallados en la velocidad de publicación, las bases de datos de revista con menor rigurosidad en su indexación son las que reflejan los tiempos de publicación más largos, como son Latindex, GSM, DOAJ, MIAR y ESCI.

La Antigüedad es otro aspecto que puede asociarse con la calidad, entendiendo que las malas revistas con el tiempo acaban desapareciendo, y sólo prevalecen las que tienen una cierta calidad y se sustentan en proyectos con una consolidación relativa a lo largo del tiempo. La mayor parte de las revistas se encuentran entre los 7 y los 18 años de antigüedad. Hay un grupo llamativo de revistas muy longevas, pero que apenas tienen presencia en bases de datos bibliográficas, como son los casos de E-Compos y Vozes e Diálogo de Brasil, Anagramas de Colombia, Contratexto de Perú; y Revista de comunicação e linguagens de Portugal. En general, las bases de datos de revistas más exigentes muestran la indexación de proyectos más consolidados.

La producción de trabajos es otro aspecto que se relaciona con la calidad y capacidad de edición de una revista. Son las revistas editadas en las bases de datos referentes de WoS las que publican un mayor número de trabajos anualmente. No obstante, están por debajo del promedio de sus respectivas áreas. Existen muchas revistas que están por debajo de los 20 artículos anuales, cuando se esperaría que fuese el mínimo si se quiere llegar a los 100 trabajos que Google Scholar requiere en el periodo de 5 años para ser incluida en Google Scholar Metrics. Son Latindex y Dialnet las bases de datos que registran un mayor número de revistas con una baja producción en 2018.

Universidades, federaciones, asociaciones, grupos de estudio, editoriales y demás responsables de la producción científica, son llamados a trabajar arduamente en la visibilización de los trabajos producidos en los países de América Latina y el Caribe, donde adicionalmente se deben tener en cuenta la periodicidad y el cumplimiento de indexación. En definitiva, las revistas iberoamericanas de Comunicación deben aunar esfuerzos por fortalecer sus niveles de producción científica, permitiéndoles, por un lado, entrar en la discusión internacional de la ciencia y el conocimiento; y por otro, penetrar las bases de datos de alto impacto, tratándose no únicamente del aumento en el número de revistas producidas sino de lograr el registro en aquellas bases con parámetros de calidad más exigentes.

Las limitaciones que se encuentran en este trabajo se centran en el análisis de la producción de trabajos científicos que se limita al último año. Así mismo, se dejaron revisitas por fuera del análisis, pues no se encontraban de forma regular en los 
portales web donde reposan o estaban únicamente en versión impresa. Para futuros estudios se recomienda hacer un análisis del contenido de los artículos que hacen parte de las bases de datos estudiadas, para medir así la presencia, el impacto y la trayectoria académica de autores y teorías de la comunicación a partir de la producción científica en las revistas iberoamericanas de comunicación. Así mismo, desde los hallazgos se puede hacer un análisis detallado sobre de los criterios que actualmente tienen las bases de datos para la indexación de las revistas, que se puede además comparar con los criterios establecidos por las mismas publicaciones.

La contribución específica al conocimiento de esta investigación radica en comprender las revistas científicas de Comunicación, un factor esencial en el campo de la producción científica en el área de la Comunicación iberoamericana, que a pesar de la importancia que tiene este tema en la academia internacional, hoy no son muchas las investigaciones en regiones como América Latina que se han encargado de identificar y analizar las características de las publicaciones del campo de la Comunicación en el actual contexto de la internacionalización del conocimiento.

A través de los resultados presentados, los investigadores del área de la comunicación podrán utilizar los indicadores para medir y analizar la presencia de las revistas científicas en las diferentes bases de datos bibliográficas. Además, esta inves-

\section{REFERENCIAS}

Alonso Gamboa, J. O.; Espinosa Reyna, F. R. (2012). La revista académica iberoamericana en Latindex. Una visión de 15 años. Revista Biblioteca Universitaria, 15(2), 123-138. https://doi.org/10.22201/dgb.0187750xp.2012.2.27

Alonso Gamboa, J. O.; Reyna Espinosa, F. R. (2015). Revistas académicas mexicanas: Panorama y prospectiva. CIENCIA ergo-sum: revista científica multidisciplinaria de la Universidad Autónoma del Estado de México, 22(3), 181-191.

Ayllón, J. M.; Martín-Martín, A.; Orduña-Malea, E.; Delgado-López-Cózar, E. (2015). Índice $H$ de las revistas científicas españolas según Google Scholar Metrics (2010-2014). EC3 Reports No. 13. Granada.

Ayllón Millán, J. M.; Martín-Martín, A.; Orduña-Malea, E.; Delgado López-Cózar, E. (2016). Índice $H$ de las revistas científicas españolas según Google Scholar Metrics (2011-2015).

Ayllón Millán, J. M.; Ruiz Pérez, R.; Delgado-López-Cózar, E. (2014). Índice $H$ de las Revistas Científicas Españolas según Google Scholar Metrics (2008-2012). EC3 Reports No. 7. Granada. tigación permitirá que revistas científicas que pertenecen a regiones como América Latina o Centro América, tengan una radiografía que se convierta en un insumo para el fortalecimiento de sus procesos de producción académica, que esté pensada desde su propia cosmovisión, y no de manera exclusiva como un camino hacia alcanzar los criterios que han venido imponiendo los sistemas hegemónicos mundiales de publicación.

\section{AGRADECIMIENTOS}

Queremos agradecer a los evaluadores del artículo por sus aportes y comentarios.

Los resultados de este artículo hacen parte del proyecto "Abordaje del pensamiento comunicacional latinoamericano en revistas científicas", financiado con recursos del Fondo Nacional de Financiamiento para la Ciencia, la Tecnología e Innovación (FCTeI) del Sistema General de Regalías (SGR).

\section{ACKNOWLEDGEMENTS}

We would like to thank the reviewers of the article for their contributions and comments.

The results of this article are part of the project "Abordaje del pensamiento comunicacional latinoamericano en revistas científicas", founded with resources from the Fondo Nacional de Financiamiento para la Ciencia, la Tecnología e Innovación (FCTeI), of the Sistema General de Regalías (SGR).

Beigel, F. (2013a). David y Goliath. El sistema académico mundial y las perspectivas del conocimiento producido en la periferia. Revista Pensamiento Universitario, (15), 1-18.

Beigel, F. (2013b). The politics of Academic Autonomy in Latin America. London: Ashgate.

Beigel, F. (2014). Publishing from the periphery: Structural heterogeneity and segmented circuits. The evaluation of scientific publications for tenure in Argentina's CONICET. Current Sociology, 62(5), 743-765. https:// doi.org/10.1177/0011392114533977

Beigel, F. (2016). Científicos Periféricos, entre Ariel y Calibán. Saberes Institucionales y Circuitos de Consagración en Argentina: Las Publicaciones de los Investigadores del CONICET. Revista Dados, 59(4), 215-255. https://doi.org/10.1590/001152582017136

Bermejo-Berros, J. (2014). Evolución de los paradigmas, metodologías y campos de la comunicación en Revista Latina de Comunicación Social durante la década 2004-2013. Revista Latina de Comunicación Social, (69), 330-353. https://doi.org/10.4185/RLCS-20141014 
Bryant, J.; Miron, D. (2004). Theory and research in mass communication. Journal of Communication, 54(4), 662-704. https://doi.org/10.1111/j.1460-2466.2004. tb02650.x

Buela-Casal, G.; López, W. (2005). Evaluación de las revistas científicas iberoamericanas de psicología. Iniciativas y estado actual. Revista Latinoamericana de Psicología, 37(1), 211-217.

Chaviano, G. (2007). Análisis bibliométrico y de calidad de la revista Signo y Pensamiento (1982-2006). Revista Signo y Pensamiento, XXVI(50), 22-32.

Corona Berkin, S. (2017). Flujos metodológicos desde el Sur latinoamericano . La zona de la comunicación y las Metodologías Horizontales. Comunicación y sociedad, (30), 69-106. https://doi.org/10.32870/cys.v0i30.6819

De-Filippo, D. (2013). La producción científica española en Comunicación en WOS . Las revistas indexadas en SSCI (2007-12). Revista Científica de Educomunicación, XXI(41), 25-34. https://doi.org/http://dx.doi. org/10.3916/C41-2013-02

Delgado-López-Cózar, E.; Martín-Martín, A. (2019). Índice $H$ de las revistas científicas españolas según Google Scholar Metrics (2014-2018). EC3 Reports. Granada. https://doi.org/10.13140/RG.2.2.36649.13923

Delgado-López-Cózar, E.; Martín-Martín, A.; OrduñaMalea, E. (2017). Índice H de las Revistas Científicas Españolas según Google Scholar Metrics (2012-2016). EC3 Reports No. 20. Granada.

Delgado, E.; Repiso, R. (2013). El impacto de las revistas de comunicación: comparando Google Scholar Metrics, Web of Science y Scopus. Comunicar, XXI(41), 45-52. https://doi.org/10.3916/C41-2013-04

Delgado López-Cózar, E.; Ayllón-Millán, J. M.; Ruiz-Pérez, R. (2013a). Índice $H$ de las revistas científicas españolas según Google Scholar Metrics (2007-2011) H Index of scientific journals published in Spain according to Google Scholar Metrics. EC3 Reports No. 3. Granada.

Delgado López-Cózar, E.; Cartagena, D. M.; Jiménez Contreras, E.; Ruiz-Pérez, R. (2013b). Índice H de las revistas españolas de Ciencias Sociales y Jurídicas según Google Scholar (2002-2011). EC3 Reports No. 4. Granada.

Delgado López-Cózar, E.; Martín-Martín, A. (2018). Índice $H$ de las Revistas Científicas Españolas según Google Scholar Metrics (2013-2017). EC3 Reports. Granada.

Devís-Devís, J.; Villamón, M.; Valenciano, J. (2014). Revistas iberoamericanas de Educación Física/Ciencias del Deporte presentes en Web of Science: evaluación y desafíos. Revista Brasileira de Ciências do Esporte, 36(4), 723-732. https://doi.org/10.1016/j. rbce. 2014.11 .004

García Aretio, L. (2017). Revistas españolas de Educación, clasificadas como excelentes por FECYT y ordenadas según el ICDS de MIAR. Recuperado de http:// blogcued.blogspot.com/2017/02/revistas-espanolasde-educacion.html
Gómez-Rodríguez, G.; Enrique Morrel, A.; Gallo-Estrada, C. (2017). A 30 años de Comunicación y Sociedad: cambios y permanencias en el campo académico de la comunicación. Comunicación y sociedad, (30), 17-44. https://doi.org/10.32870/cys.v0i30.6838

Klein, D.; Chiang, E. (2004). The Social Science Citation Index: A Black Box - with an Ideological Bias? Econ Journal Watch, 1(1), 134-165.

Liberatore, G.; Herrero-Solana, V. (2009). Caracterización temática de la investigación en Ciencia de la Información en Brasil en el período 2000-2009 from 20002009. TransInformação, 25(3), 225-235. https://doi. org/10.1590/S0103-37862013000300005

López -Robles, J.-R.; Guallar, J.; Otegui-Olaso, J. R.; Gamboa-Rosales, N.-K. (2019). El profesional de la información (EPI): Bibliometric and thematic analysis (2006-2017). El Profesional de la Información, 28(4), 1-23. https://doi.org/https://doi.org/10.3145/ epi.2019.jul.17

Maldonado, A. (2017). Contenido y uso de DOAJ. https:// doi.org/10.13140/RG.2.2.20307.48165

Martín-Martín, A.; Orduna-Malea, E.; Delgado LópezCózar, E. (2017). Journal Scholar Metrics: building an Arts, Humanities, and Social Sciences journal ranking with Google Scholar data. En 22nd International Conference on Science, Technology y Innovation Indicators (STI). Granada. https://doi.org/10.17605/OSF. IO/VXNW6

Martínez-Nicolás, M.; Sapera, E.; Carrasco-Campos, Á. (2018). La investigación sobre comunicación en España en los últimos 25 años (1990-2014). Objetos de estudio y métodos aplicados en los trabajos publicados en revistas españolas especializadas. Revista de Metodología de Ciencias Sociales, (42), 37-69. https://doi. org/10.5944/empiria.42.2019.23250

Mazo Flórez, C. E. (2017). Buenas prácticas editoriales de revistas de Ciencias Sociales de acceso abierto en América Latina. Universidad de Antioquia.

Mosbah-Natanson, S.; Gingras, Y. (2015). The globalization of social sciences? Evidence from a quantitative analysis of 30 years of production, collaboration and citations in the social sciences (1980-2009). Current Sociology, 62(5), 626-646. https://doi. org/10.1177/0011392113498866

Murray, D.; Chabot, C. (2013). Tableau Your Data!: Fast and Easy Visual Analysis with Tableau Software. John Wiley y Sons Inc.

Ortíz, R. (2009). La supremacía del inglés en las ciencias sociales. Buenos Aires: Siglo Veintiuno Editores.

Pérez-Rodríguez, A.; García-Ruíz, R.; Aguaded, I. (2018). Comunicar: calidad, visibilización e impacto. Revista Española de Pedagogía, 76(271), 481-498. https:// doi.org/10.22550/REP-3-2018-05

Picco, P.; Aguirre-Liguera, N.; Maldini, J.; Simón, L.; Petroccelli, P.; Fontans, E.; Fager, J.; Ceretta, M. G. (2010). La comunicación científica en Uruguay: es- 
tudio de las publicaciones de los investigadores activos del Sistema Nacional de Investigadores (20092010). TransInformação, 26(2), 155-165. https://doi. org/10.1590/0103-37862014000200005

Piñeiro-Naval, V.; Morais, R. (2019). Estudio de la producción académica sobre comunicación en España e Hispanoamérica. Comunicar, XXVII(61), 113-123. https://doi.org/10.3916/C61-2019-10

Pivatto Brum, W. ; Sildo da Cunha, J.; Gressler Pianezzola, V. H. (2016). A Revista Perspectivas em Ciência da Informação e seu panorama científico no período 2010 a 20. Perspectivas em Ciência da Informação, 21(3), 204-221. https://doi.org/10.1590/1981-5344/2651

Repiso Caballero, R.; Delgado López-Cózar, E. (2014). H Index Communication Journals according to Google Scholar Metrics (2009-2013). EC3 Reports 10.

Repiso, R. (2015). Cómo identificar una revista de calidad. Cardiocore, 50(2), 46-48. https://doi.org/10.1016/j. carcor.2014.12.002

Repiso, R.; Jiménez-contreras, E.; Aguaded, I. (2017). Revistas Iberoamericanas de Educación en SciELO Citation Index y Emerging Source Citation Index. Revista Española de Documentación Científica, 40(4), e186. https://doi.org/10.3989/redc.2017.4.1445

Repiso, R.; Orduña-Malea, E.; Aguaded, I. (2019). Revistas científicas editadas por universidades en Web of Science: características y contribución a la marca universidad. El Profesional de la Información, 28(4), e280405. https://doi.org/10.3145/epi.2019.jul.05

Repiso, R.; Torres, D. (2016). Características e implicaciones de la base de datos Emerging Sources Citation Index (Thomson Reuters): las revistas en estado transitorio. AnuarioThinkEPI, 10, 234-236. https://doi. org/10.3145/thinkepi.2016.46
Rodríguez-Gairín, J.-M.; Somoza-Fernández, M.; Urbano, C. (2011). Hacia un entorno colaborativo de editores, autores y evaluadores de revistas. El Profesional de la Informacion, 20 (5), 589-595. https://doi. org/10.3145/epi.2011.sep.15

Rodríguez, A. J.; Ochoa, S.; Mayta, P. (2014). Impacto de las revistas de salud colombianas: comparación de Publindex versus Google Scholar Metrics, SciELO y SCOPUS. Revista Cubana de Información en Ciencias de la Salud, 25 (1), 24-35.

Rogel-Salazar, R.; Santiago-Bautista, I.; Martínez-Dominguez, N. (2017). Revistas científicas latinoamericanas de Comunicación indizadas en WoS, Scopus y bases de datos de Acceso Abierto. Comunicación y sociedad, (30), 167-196. https://doi.org/10.32870/cys.v0i30.6514

Romero Peña, A.; Iturriaga Barco, D.; Sáenz de Pablo, J. (2015). DIALNET: un proyecto local de éxito global. Una mirada crítica a su desarrollo. En Pensar con Historia desde el Siglo XXI, pp. 5933-5946. Madrid: Universidad Autónoma de Madrid.

Torre-Espinosa, M.; Repiso, R.; Montero Díaz, J. (2019). Factor de Impacto y comportamiento bibliométrico de las revistas de "Film, Radio y Television" de Web of Science. Revista Española de Documentación Científica, 42(3), e243. https://doi.org/10.3989/ edc. 2019.3 .1630

Walter, N.; Cody, M. J.; Ball-Rokeach, S. J. (2018). The Ebb and Flow of Communication Research: Seven Decades of Publication Trends and Research Priorities. Journal of Communication, 68(2), 424-440. https:// doi.org/10.1093/joc/jqx015

WSSR-Unesco. (2015). World Science Report. París. 